\title{
RELATIVE FROBENIUS OF PLANE SINGULARITIES
}

\author{
D. DAIGLE
}

\begin{abstract}
In view of the well-known conjecture concerning the classification of lines in the affine plane in characteristic $p>0$, it is desirable to understand how the characteristic pairs of an irreducible algebroid plane curve are affected by the relative Frobenius. This paper determines the relation between the characteristic sequences $[x, y]$ and $\left[x, y^{p}\right]$, where $x$ and $y$ are formal power series in one variable with coefficients in a field of characteristic $p>0$.
\end{abstract}

Throughout this paper, $\mathbf{k}$ is an algebraically closed field of characteristic $p>0, t$ is an indeterminate over $\mathbf{k}$ and $\mathbf{k}((t))$ is the field of fractions of the ring $\mathbf{k}[[t]]$ of formal power series in $t$. An affine plane curve with one place at infinity may be thought of as an epimorphism $\varepsilon: \mathbf{k}[X, Y] \rightarrow A$, where $A$ is a one dimensional domain contained in all but one (say $A \nsubseteq R$ ) valuation rings of $q t(A) / \mathbf{k}$. Note that if $x=\varepsilon(X)$ and $y=\varepsilon(Y)$ then $\varepsilon^{\prime}: \mathbf{k}[X, Y] \rightarrow A^{\prime}=$ $\mathbf{k}\left[x, y^{p}\right]$ defined by $\varepsilon^{\prime}(X)=x$ and $\varepsilon^{\prime}(Y)=y^{p}$ is also an affine plane curve with one place at infinity. We say that $\varepsilon^{\prime}$ is obtained from $\varepsilon$ by performing the "relative Frobenius" operation. This paper is part of an attempt to answer:

Question 1. How is the singularity at infinity affected by the relative Frobenius?

As a motivation for this question we recall the well-known conjecture which asserts that every affine plane curve biregular to the affine line can be obtained from a line of degree one by performing a finite sequence of operations, each operation being either an automoprhism of the plane or the relative Frobenius, the latter being allowed only if $A=A^{\prime}$. (See $[1,2,4,5]$.)

Let the notation be as above. Then the completion of $R$ may be identified with $\mathbf{k}[[t]]$ and as a result $x, y \in \mathbf{k}((t))$ have negative orders (unless $x \in \mathbf{k}$ or $y \in \mathbf{k}$, which is a trivial case). Define $x_{\infty}, y_{\infty} \in t \mathbf{k}[[t]]$ by

$$
\left(x_{\infty}, y_{\infty}\right)= \begin{cases}(1 / x, y / x), & \text { if ord } x<\text { ord } y, \\ (1 / y, x / y), & \text { if ord } x>\text { ord } y, \\ (1 / x, y / x-\alpha), & \text { if ord } x=\text { ord } y, \text { and } \\ & \alpha=\text { constant term of } y / x .\end{cases}
$$

Received by the editors March 30, 1989.

1980 Mathematics Subject Classification (1985 Revision). Primary 14H20, 14E25.

Key words and phrases. Frobenius, characteristic pairs, embeddings of lines.

Partly supported by a fellowship from NSERC Canada. 
Then a description of the singularity of $\varepsilon$ at infinity is given by the characteristic sequence [ $x_{\infty}, y_{\infty}$ ] of $x_{\infty}$ and $y_{\infty}$, as defined in [3] or [6]. On the other hand let $\left(x^{\prime}, y^{\prime}\right)=\left(x, y^{p}\right)$ and consider $\left(x_{\infty}^{\prime}, y_{\infty}^{\prime}\right)$. A more precise version of question 1 is

Question 2. How are $\left[x_{\infty}, y_{\infty}\right]$ and $\left[x_{\infty}^{\prime}, y_{\infty}^{\prime}\right]$ related?

In [3 and 6], the characteristic sequence of a pair of series is defined only in the case where the two series have positive orders. In the first section, we extend the definition to a larger set of pairs of series and (1.4) gives a very simple relation between $\left[x_{\infty}, y_{\infty}\right]$ and $[x, y]$. In view of that, it is clear that question 2 is equivalent to

Question 3. How are $[x, y]$ and $\left[x, y^{p}\right]$ related?

The third section is devoted to the main result, Theorem 3.1, which answers question 3 for $x, y \in \mathbf{k}((t))^{*}$ of nonzero orders and such that $\mathbf{k}\left(x, y^{p}\right)$ is dense in $\mathbf{k}((t))$. Note that the case where $\mathbf{k}\left(x, y^{p}\right)$ is not dense in $\mathbf{k}((t))$ is an easy corollary of (3.1), but does not appear here. The second section develops the notions needed for stating and proving the theorem.

I would like to thank A. Sathaye for innumerable discussions about problems related to this work.

Notations. The set of integers (resp. positive integers) is denoted $\mathbf{Z}$ (resp. $\mathbf{N}$ ). A subset $H$ of $\mathbf{Z}$ is said to be convex if $j \in H$ whenever $i<j<k$ and $i, k \in H$. If $x=\left(\ldots, x_{i-1}, x_{i}, x_{i+1}, \ldots\right)$ is a sequence of integers then $\left(\ldots, x_{i-1}, \hat{x}_{i}, x_{i+1}, \ldots\right)$ is the sequence obtained from $x$ by omitting the term $x_{i}$ if and only if $x_{i}=0$. In general, we adopt the notation used in [3]. In particular, if $a \in \mathbf{Z} \backslash\{0\}$ then

$$
|a|=|a|_{p}=\max \left\{p^{\nu} \mid \nu \geq 0 \text { is an integer and } p^{\nu} \mid a\right\}
$$

and $a_{0}=a /|a|$. The ordinary absolute value will never be used, so no confusion is possible.

If $x \in \mathbf{k}((t))^{*}=\mathbf{k}((t)) \backslash\{0\}$ then $x=\sum a_{i} t^{i}$ for some family $\left(a_{i}\right)_{i \in \mathbf{Z}}$ of elements of $\mathbf{k}$, the set $\operatorname{Supp}_{t} x=\left\{i \mid a_{i} \neq 0\right\}$ is bounded below and its minimum is denoted ord $x$. The valuation "ord" determines a topology on $\mathbf{k}((t))$ and we denote by $\bar{K}$ the closure of a subset $K$ of $\mathbf{k}((t))$.

\section{Characteristic Sequences}

In this section we define the characteristic sequence $[x, y]$ for any $x, y \in$ $\mathbf{k}((t))^{*}$ of nonzero orders, and we state some basic properties of those sequences. We would like to acknowledge the fact that most of the material in this section is obtained from Moh's paper [3] simply by dropping his assumptions on $x$ and $y$ and making some straightforward adaptations in his proofs. Consequently most proofs are omitted. Note that only the definitions and some properties given in Moh's paper are generalized here, not his results dealing specifically with algebroid plane curves. 


\section{Definitions 1.1.}

1. If $S \subset \mathbf{Z}$ is bounded below and $d \in \mathbf{N}$, let $S(d)=\min (S \backslash d \mathbf{Z}) \quad(=\infty$ if $S \backslash d \mathbf{Z}=\varnothing)$.

2. Let $S$ be a nonempty subset of $\mathbf{Z}$, bounded below, and let $c \in \mathbf{N}$. Then an integer $k$ and a finite sequence of integers $\langle S ; c\rangle=\left(q_{1}, \ldots, q_{k}\right)$ are defined inductively as follows, together with another sequence $\left(d_{2}, \ldots\right.$, $\left.d_{k+1}\right)$ :

- $q_{1}=\min S$ and $d_{2}=\operatorname{gcd}\left(c, q_{1}\right)$;

- if for some $i \geq 1,\left(q_{1}, \ldots, q_{i}\right)$ and $\left(d_{2}, \cdots, d_{i+1}\right)$ are already defined then let

$$
\begin{array}{ll}
\left.\begin{array}{l}
q_{i+1}=S\left(d_{i+1}\right)-\left(q_{1}+\cdots+q_{i}\right) \\
d_{i+2}=\operatorname{gcd}\left(d_{i+1}, q_{i+1}\right)
\end{array}\right\} & \text { if } S\left(d_{i+1}\right)<\infty, \\
k=i, & \text { if } S\left(d_{i+1}\right)=\infty .
\end{array}
$$

3. Suppose $x, y \in \mathbf{k}((t))^{*}$, ord $x=m \neq 0$, ord $y=n \neq 0$ and $|m| \leq|n|$. Choose $x_{1} \in \mathbf{k}[[t]]$ of order $|m|$ such that $x_{1}^{m_{0}}=x$. Then there is a unique expression

$$
y=\sum_{i \geq n /|m|} a_{i} x_{1}^{i}+\rho
$$

such that $a_{i} \in \mathbf{k}, \rho \in \mathbf{k}((t))$, and if $\rho \neq 0$ then $|\operatorname{ord} \rho|<|m|$ and

$$
a_{i} \neq 0 \Rightarrow i|m|<\operatorname{ord} \rho \text {. }
$$

Such expressions will be referred to as expressions satisfying the conditions of (1.1.3). Note that $\rho=0$ if and only if $y \in \mathbf{k}\left(\left(x_{1}\right)\right)$, if and only if $\mathbf{k}(x, y) \subset \mathbf{k}\left(\left(x_{1}\right)\right)$.

4. Suppose $x, y \in \mathbf{k}((t))^{*}$, ord $x=m \neq 0$, ord $y=n \neq 0$, and $|m| \leq$ $|n|$, and let $c \in \mathbf{N}$ be such that $c \mid \operatorname{gcd}(m, n)$ and $|c|=|m|$. Then the $c$-sequence $[x, y: c]=\left(q_{1}, \ldots, q_{h}\right)$ of $x, y$ with respect to $c$ is defined inductively as follows. Let

$$
y=\sum_{i \geq n /|m|} a_{i} x_{1}^{i}+\rho
$$

be an expression satisfying the conditions of (1.1.3) and write

$$
\left\langle|m| \operatorname{Supp}_{x_{1}}(y-\rho) ; c\right\rangle=\left(q_{1}, \ldots, q_{k}\right) .
$$

- If $\rho=0$ then set $h=k$ and $[x, y: c]=\left(q_{1}, \ldots, q_{h}\right)$;

- if $\rho \neq 0$ then set $[x, y: c]=\left(q_{1}, \ldots, q_{k}, \ldots, q_{h}\right)$ where $q_{k+1}$, $\ldots, q_{h}$ are given by

$$
\begin{aligned}
q_{k+1} & =\operatorname{ord} \rho-\left(q_{1}+\cdots+q_{k}\right), \\
{\left[y_{1}, x: \bar{c}\right] } & =\left(m, q_{k+2}, \ldots, q_{h}\right),
\end{aligned}
$$

where $\bar{c}=\operatorname{gcd}\left(c, q_{1}, \ldots, q_{k+1}\right)$ and $y_{1}=\rho / x_{1}^{n /|m|}$. 
Proposition 1.2. Suppose $x, y \in \mathbf{k}((t))^{*}$, ord $x=m \neq 0$, ord $y=n \neq 0$, and $|m| \leq|n|$, and let $c \in \mathbf{N}$ be such that $c \mid \operatorname{gcd}(m, n)$, and $|c|=|m|$. Write $[x, y: c]=\left(q_{1}, \ldots, q_{h}\right)$.

1. If $d \in \mathbf{Z} \backslash\{0\}$ and $|d|=1$, then $\left[x^{d}, y: c\right]=[x, y: c]$.

2. If $d \in \mathbf{Z} \backslash\{0\}$ and $|d|=1$, then $\left[x, y^{d}: c\right]=\left(d q_{1}, q_{2}, \ldots, q_{h}\right)$.

3. (Inversion Theorem) If $|m|=|n|$, then $[y, x: c]=\left(m, q_{2}, \ldots, q_{h}\right)$.

4. If $m \neq n$, then $[x, y / x: c]=\left(q_{1}-m, q_{2}, \ldots, q_{h}\right)$.

5. If $m \neq n$ and $|m-n| \leq|n|$, then $[x / y, y: c]=[x, y: c]$.

6. If $y=\sum a_{i} x_{1}^{i}+\rho$ is an expression satisfying the conditions of (1.1.3) and $\rho \neq 0$ then, in the definition of $[x, y: c]$ given in (1.1), the integers $q_{k+2}, \ldots, q_{h}$ can also be defined by

$$
[\rho, x: \bar{c}]=\left(m, q_{k+2}, \ldots, q_{h}\right) \text {. }
$$

Proof. The first five assertions follow from [3]. More precisely, (1) and (2) are obtained by adapting the proof of Proposition 1; (3) follows from the proof of Theorem 2; (4) and (5) are obtained by imitating the proof of Proposition 2.

We prove (6). Let $e=\operatorname{gcd}\left(m, n, q_{2}, \ldots, q_{k}\right)$ and note that $|e|=|m|$. So ord $z=e$, where $z=x_{1}^{e_{0}}$. Let us write

$$
\left[y_{1}, x: \bar{c}\right]=\left(m, q_{k+2}, \ldots, q_{h}\right) \text {. }
$$

Since $z^{d}=x$ for some $d \in \mathbf{Z} \backslash\{0\}$ with $|d|=1$, and since $\bar{c} \mid \operatorname{gcd}\left(\operatorname{ord} y_{1}\right.$, ord $z$ ) and $|\bar{c}|=\left|\operatorname{ord} y_{1}\right|$, we have by part (2)

$$
\left[y_{1}, z: \bar{c}\right]=\left(e, q_{k+2}, \ldots, q_{h}\right) .
$$

Let $r \in \mathbf{Z}$ be such that $z^{r}=x_{1}^{n /|m|}$. Then by $\pm r$ applications of part (5) we get

$$
[\rho, z: \bar{c}]=\left(e, q_{k+2}, \ldots, q_{h}\right) .
$$

Since $x=z^{d}$, the desired result follows from this and part (2).

Definition 1.3. Suppose $x, y \in \mathbf{k}((t))^{*}$, ord $x=m \neq 0$ and ord $y=n \neq 0$. Then the characteristic sequence of $x$ and $y$ is $[x, y]=\left(m, n, q_{2}, \ldots, q_{h}\right)$, where $q_{2}, \ldots, q_{h}$ are given by

$$
\begin{array}{ll}
{[x, y: c]=\left(n, q_{2}, \ldots, q_{h}\right),} & \text { if }|m| \leq|n|, \\
{[y, x: c]=\left(m, q_{2}, \ldots, q_{h}\right),} & \text { if }|n| \leq|m|,
\end{array}
$$

where $c=\operatorname{gcd}(m, n)$. Note that the inversion Theorem 1.2 guarantees that this is well defined.

Remark. One can see that if $x, y \in \mathbf{k}((t))^{*}$ have nonzero orders and $[x, y]=$ $\left(m, n, q_{2}, \ldots, q_{h}\right)$ then

$$
[\mathbf{k}((t)): \overline{\mathbf{k}(x, y)}]=\operatorname{gcd}\left(m, n, q_{2}, \ldots, q_{h}\right) .
$$

The following is an easy consequence of (1.2). It relates questions 2 and 3 , in the introduction. 
Lemma 1.4. Let $x, y \in \mathbf{k}((t))^{*}$, ord $x=m \neq 0$, ord $y=n \neq 0, y / x \notin \mathbf{k}$ and $[x, y]=\left(m, n, q_{2}, \ldots, q_{h}\right)$. Define $x_{\infty}, y_{\infty} \in \mathbf{k}((t))^{*}$ as in the introduction and let $m_{\infty}=\operatorname{ord} x_{\infty}$ and $n_{\infty}=\operatorname{ord} y_{\infty}$. Then

$$
\left[x_{\infty}, y_{\infty}\right]= \begin{cases}\left(m_{\infty}, n_{\infty}, q_{2}, \ldots, q_{h}\right), & \text { if } m \neq n, \\ \left(m_{\infty}, n_{\infty}, q_{2}-n_{\infty}, q_{3}, \ldots, q_{h}\right), & \text { if } m=n \text { and } m \mid n_{\infty}, \\ \left(m_{\infty}, n_{\infty}, q_{3}, \ldots, q_{h}\right), & \text { if } m=n \text { and } m \nmid n_{\infty} .\end{cases}
$$

Moreover, if $m=n$ and $m+n_{\infty}$ then $n_{\infty}=q_{2}$.

\section{Abstract SEQuenCES}

This section defines abstract versions of the $c$-sequence $[x, y: c]$ and the characteristic sequence $[x, y]$ of a pair of power series. Those abstract sequences are called $Q$-sequences and abstract characteristic sequences respectively. A mapping $\Phi_{Q}$ is defined in such a way that $\Phi_{Q}(q, \mathscr{D})$ is a $Q$-sequence whenever $q$ is a $Q$-sequence and $\mathscr{D}$ belongs to a certain set $\operatorname{Part}(q)$ determined by $q$. Corresponding notions are defined for abstract characteristic sequences.

\section{Definitions 2.1.}

1. A $Q$-sequence is a triple $q=\left(c ; m ;\left(q_{i}\right)_{i \in H}\right)$ such that

- $H=\{a, \ldots, h\}$ is a nonempty, finite, convex subset of $\mathbf{Z}$ and $\left(q_{i}\right)_{i \in H}$ is a sequence indexed by $H$;

- $c \in \mathbf{N}, m, q_{a} \in \mathbf{Z} \backslash\{0\}$ and $q_{i} \in \mathbf{N}, a<i \leq h$;

- $c \mid \operatorname{gcd}\left(m, q_{a}\right)$ and $|c|=|m|$;

- if we define $d_{a+1}=c$ and $d_{i+1}=\operatorname{gcd}\left(d_{i}, q_{i}\right), a<i \leq h$, then $d_{a+1}>\cdots>d_{h+1}=1$.

Then $d_{a+1}, \ldots, d_{h+1}$ are called the gcd's of $q$. When we wish to emphasize that the indexing set is $H$, we say that $q$ is a $Q$ sequence on $H$.

For the rest of these definitions, let $q=\left(c ; m ;\left(q_{i}\right)_{i \in H}\right)$ be a $Q$-sequence on $H=\{a, \ldots, h\}$, with gcd's $d_{a+1}, \ldots, d_{h+1}$.

2. $L(q)=\{h\} \cup\left\{i \in H \mid a<i<h\right.$ and $\left.\left|d_{i}\right|>\left|d_{i+1}\right|\right\}$.

3. If $\mathscr{S}$ is a (possibly empty) collection of pairwise disjoint subsets of $H$, let $\mathscr{S}^{*}$ be the collection of maximal convex subsets of $H \backslash \bigcup \mathscr{S}$. We say that $\mathscr{S}$ is q-allowable if the following conditions are satisfied:

- Either $a \in \bigcup \mathscr{S}$ or $|m|<\left|q_{a}\right|$;

- every $S \in \mathscr{S}$ is nonempty, convex and satisfies $S \cap L(q)=$ $\{\max S\}$;

- every $S \in \mathscr{S}^{*}$ satisfies (where $j=\max S$ ) $S \cap L(q) \subseteq\{j\}$ and $i \in S \backslash\{j\} \Rightarrow\left|q_{i}\right|>\left|d_{j}\right|$. 
4. If $\mathscr{S}$ is $q$-allowable then let $J(q, \mathscr{S}) \subseteq H$ be the set

$$
\begin{aligned}
& \left\{i \in \bigcup \mathscr{S} \mid \exists_{S \in \mathscr{S}}(i+1=\min S) \text { or }\left(a<i=h \text { and }\left|d_{h}\right|>1\right)\right\} \\
& \quad \cup\left\{i \in \bigcup \mathscr{S}^{*}|| q_{i}|>| d_{i+1} \mid \text { and }\left(\exists_{S \in \mathscr{S}}(i+1=\min S) \text { or } i=h\right)\right\} .
\end{aligned}
$$

5. $\operatorname{Part}(q)$ is the set of all pairs $\mathscr{D}=(\mathscr{S}, f)$ where $\mathscr{S}$ is a $q$-allowable collection of subsets of $H$ and $f: H \rightarrow \mathbf{Z}$ satisfies

- $f(i)=0$, if $i \notin J(q, \mathscr{S})$;

- if $i \in J(q, \mathscr{S})$ then $f(i)>0$ and $\operatorname{gcd}\left(p d_{i+1}, f(i)\right)=d_{i+1}$; if moreover $i<h$ then $f(i)<q_{i+1}$.

In order to be able to make inductive arguments, we have to define a notion of restriction of a pair $(q, \mathscr{D})$, where $q$ is a $Q$-sequence and $\mathscr{D} \in \operatorname{Part}(q)$.

Definitions 2.2. Let $q=\left(c ; m ;\left(q_{i}\right)_{i \in H}\right)$ be a $Q$-sequence on $H=\{a, \ldots, h\}$, with gcd's $d_{a+1}, \ldots, d_{h+1}$.

1. If $|m|>1$, the restriction of type 1 of $q$ is the sequence

$$
\tilde{q}=\left(d_{j+1} ; q_{a}+\cdots+q_{j} ; m, q_{j+1}, \ldots, q_{h}\right),
$$

where $j=\min L(q)$. Note that $a<j \leq h$.

2. If $\left|q_{a}\right|>|m|$ then a restriciton of type 2 of $q$ is a sequence of the form ${ }^{1}$

$$
\tilde{q}=\left(d_{j+1} ; q_{a}+\cdots+q_{j}+\alpha ; m, q_{j+1}-\alpha, q_{j+2}, \ldots, q_{h}\right),
$$

where $j \in H$ and $\alpha \in \mathbf{Z}$ satisfy

- $\left|q_{i}\right|>|m|$ for $a \leq i<j$,

- $\alpha=0$, if $\left|q_{j}\right|=\left|d_{j+1}\right|$,

- $\alpha>0$ and $\operatorname{gcd}\left(p d_{j+1}, \alpha\right)=d_{j+1}$, if $\left|q_{j}\right|>\left|d_{j+1}\right|$; if moreover $j<h$ then $\alpha<q_{j+1}$.

3. A restriction of $q$ is a sequence $\tilde{q}$ which is either the restriction of type 1 or a restriction of type 2 of $q$. Any restriction $\tilde{q}=\left(d_{j+1} ; \ldots\right)$ of $q$ is a $Q$-sequence on $\{j, \ldots, h\}$ and satisfies $L(\tilde{q})=(L(q) \backslash\{j\}) \cup\{h\}$. It may happen that a restriction of $q$ be of types 1 and 2 .

4. $\operatorname{Res}(q)$ denotes the set of all pairs $(\tilde{q}, \widetilde{D})$ such that $\tilde{q}$ is a restriction of $q, \widetilde{\mathscr{D}}=(\tilde{\mathscr{S}}, \tilde{f}) \in \operatorname{Part}(\tilde{q})$, and either $\tilde{q}$ is of type 1 and $j \in \bigcup \widetilde{\mathscr{S}}^{*}$, or $\tilde{q}$ is of type 2 and $j \in \bigcup \widetilde{\mathscr{S}}$, where $j \in H$ is such that $\tilde{q}=\left(d_{j+1} ; \ldots\right)$.

5. Let $\mathscr{D}=(\mathscr{S}, f) \in \operatorname{Part}(q)$. The pair $(q, \mathscr{D})$ is minimal if $|m|=1$ and $\mathscr{S}=\{H\}$.

6. Let $\mathscr{D}=(\mathscr{S}, f) \in \operatorname{Part}(q)$ be such that $(q, \mathscr{D})$ is not minimal. We define the restriction of $(q, \mathscr{D})$, denoted $\operatorname{res}(q, \mathscr{D})$, to be the pair $(\tilde{q}, \widetilde{\mathscr{D}}) \in \operatorname{Res}(q)$ where $\tilde{q}$ and $\widetilde{\mathscr{D}}$ are defined as follows. Let $S_{1}=$ $\{a, \ldots, j\}$ be the member of $\mathscr{S} \cup \mathscr{S}^{*}$ which contains $a$ and let $\widetilde{H}=$ $\{j, \ldots, h\}$.

\footnotetext{
${ }^{1}$ To be precise, $\tilde{q}=\left(1 ; q_{a}+\cdots+q_{h}+\alpha ; m\right)$, if $j=h$.
} 
- If $S_{1} \in \mathscr{S}$, let $\tilde{q}$ be the restriction of type 1 of $q$ and let $\widetilde{\mathscr{S}}=$ $\mathscr{S} \backslash\left\{S_{1}\right\}$. Then $\tilde{q}$ is a $Q$-sequence on $\widetilde{H}, \widetilde{\mathscr{S}}$ is $\tilde{q}$-allowable and $J(\tilde{q}, \widetilde{\mathscr{S}})=J(q, \mathscr{S})$. Let $\widetilde{\mathscr{D}}=(\widetilde{\mathscr{S}}, \tilde{f})$, where $\tilde{f}: \widetilde{H} \rightarrow \mathbf{Z}$ is the restriction of $f$ to $\widetilde{H}$. Then $\widetilde{\mathscr{D}} \in \operatorname{Part}(\tilde{q})$. Note that $j \in \bigcup \widetilde{\mathscr{S}}^{*}$.

- If $S_{1} \in \mathscr{S}^{*}$, note that $j \in J(q, \mathscr{S}) \Leftrightarrow\left|q_{j}\right|>\left|d_{j+1}\right|$ and let $\tilde{q}$ be the following restriction of type 2 of $q$ :

$\tilde{q}=\left(d_{j+1} ; q_{a}+\cdots+q_{j}+f(j) ; m, q_{j+1}-f(j), q_{j+2}, \ldots, q_{h}\right)$.

If $j=h$, let $\widetilde{\mathscr{S}}=\{\{h\}\}$; if $j<h$, let $\widetilde{\mathscr{S}}$ be the collection of subsets of $\widetilde{H}$ which is obtained from $\mathscr{S}$ by replacing $S_{2}$ by $S_{2} \cup\{j\}$, where $S_{2}$ is the member of $\mathscr{S}$ which contains $j+$ 1 . Note that $\tilde{q}$ is a $Q$-sequence on $\widetilde{H}, \widetilde{\mathscr{S}}$ is $\tilde{q}$-allowable and $J(\tilde{q}, \widetilde{\mathscr{S}})=J(q, \mathscr{S}) \backslash\{j\}$. Let $\widetilde{\mathscr{D}}=(\widetilde{\mathscr{S}}, \tilde{f})$, where $\tilde{f}: \widetilde{H} \rightarrow \mathbf{Z}$ is defined by $\tilde{f}(j)=0$ and $\tilde{f}(i)=f(i), j<i \leq h$. Then $\widetilde{\mathscr{D}} \in \operatorname{Part}(\tilde{q})$. Note that $j \in \bigcup \widetilde{\mathscr{S}}$.

Note that if $q$ is a $Q$-sequence and $\mathscr{D} \in \operatorname{Part}(q)$ then $\operatorname{res}^{(i)}(q, \mathscr{D})$ is minimal for some nonnegative integer $i$.

Lemma 2.3. If $q$ is a $Q$-sequence then the map

$$
\{\mathscr{D} \in \operatorname{Part}(q) \mid(q, \mathscr{D}) \text { is not minimal }\} \rightarrow \operatorname{Res}(q),
$$

defined by $\mathscr{D} \mapsto \operatorname{res}(q, \mathscr{D})$, is bijective.

The verification of 2.3 is straightforward, and omitted.

Definition 2.4. Let $q=\left(c ; m ;\left(q_{i}\right)_{i \in H}\right)$ be a $Q$-sequence on $H=\{a, \ldots, h\}$, and let $\mathscr{D}=(\mathscr{S}, f) \in \operatorname{Part}(q)$. We inductively define a sequence $\Phi_{Q}(q, \mathscr{D})$ as follows.

- If $(a, \mathscr{D})$ is minimal,

$$
\Phi_{Q}(q, \mathscr{D})=\left(c ; m ; p q_{a}, \ldots, p q_{h}\right) .
$$

- If $(q, \mathscr{D})$ is not minimal let $(\tilde{q}, \widetilde{D})=\operatorname{res}(q, \mathscr{D})$ and define $Q_{2}, \ldots, Q_{T}$ by $\Phi_{Q}(\tilde{q}, \widetilde{D})=\left(\tilde{c} ; \tilde{m} ; Q_{1}, \ldots, Q_{T}\right)$. Let $S_{1}=$ $\{a, \ldots, j\}$ be the member of $\mathscr{S} \cup \mathscr{S}^{*}$ which contains $a$, and define $\Phi_{Q}(q, \mathscr{D})$ to be the sequence

$\left(c ; m ; p q_{a}, \ldots, p q_{j-1}, p q_{j}+Q_{2}, Q_{3}, \ldots, Q_{T}\right)$, if $S_{1} \in \mathscr{S}$ and $d_{j}=p d_{j+1}$, $\left(c ; m ; p q_{a}, \ldots, p q_{j}, Q_{2}, \ldots, Q_{T}\right)$, $\left(p c ; p m ; q_{a}, \ldots, q_{j}, \widehat{f(j)}, Q_{2}, \ldots, Q_{T}\right), \quad$ if $S_{1} \in \mathscr{S}^{*}$.

Lemma 2.5. Let $q=\left(c ; m ;\left(q_{i}\right)_{i \in H}\right)$ be a $Q$-sequence on $H=\{a, \ldots, h\}$, with gcd's $d_{a+1}, \ldots, d_{h+1}$, and let $\mathscr{D}=(\mathscr{S}, f) \in \operatorname{Part}(q)$.

1. If $(q, \mathscr{D})$ is minimal then the gcd's of $\Phi_{Q}(q, \mathscr{D})$ are $d_{a+1}, \ldots, d_{h+1}$. 
2. If $(q, \mathscr{D})$ is not minimal then let $(\tilde{q}, \widetilde{D})=\operatorname{res}(q, \mathscr{D})$ and let $D_{2}, \ldots$, $D_{T+1}$ be the successive gcd's of $\Phi_{Q}(\tilde{q}, \widetilde{D})=\left(\tilde{c} ; \tilde{m} ; Q_{1}, \ldots, Q_{T}\right)$. Let also $S_{1}=\{a, \ldots, j\}$ be the member of $\mathscr{S} \cup \mathscr{S}^{*}$ that contains $a$. Then the gcd's of $\Phi_{Q}(q, \mathscr{D})$ are:

$\left(d_{a+1}, \ldots, d_{j-1}, d_{j}=D_{2}, D_{3}, \ldots, D_{T+1}\right), \quad$ if $S_{1} \in \mathscr{S}$ and $d_{j}=p d_{j+1}$, $\left(d_{a+1}, \ldots, d_{j}, p d_{j+1}=D_{2}, D_{3}, \ldots, D_{T+1}\right), \quad$ if $S_{1} \in \mathscr{S}$ and $d_{j} \neq p d_{j+1}$, $\left(p d_{a+1}, \ldots, p d_{j+1}, d_{j+1}=D_{2}, D_{3}, \ldots, D_{T+1}\right)$, if $S_{1} \in \mathscr{S}^{*}$ and $j \in J(q, \mathscr{S})$, $\left(p d_{a+1}, \ldots, p d_{j}, d_{j+1}=D_{2}, D_{3}, \ldots, D_{T+1}\right)$, if $S_{1} \in \mathscr{S}^{*}$ and $j \notin J(q, \mathscr{S})$.

The proof of (2.5) is a straightforward verification that we leave to the reader.

Corollary 2.6. If $q$ is a $Q$-sequence and $\mathscr{D} \in \operatorname{Part}(q)$ then $\Phi_{Q}(q, \mathscr{D})$ is a $Q$ sequence. To be precise, we regard $\Phi_{Q}(q, \mathscr{D})$ as a $Q$-sequence on a set whose minimium is 1 .

Lemma 2.7. If $q$ is a $Q$-sequence, $\mathscr{D}, \mathscr{D}^{\prime} \in \operatorname{Part}(q)$ and

$$
\Phi_{Q}(q, \mathscr{D})=\Phi_{Q}\left(q, \mathscr{D}^{\prime}\right)
$$

then $\mathscr{D}=\mathscr{D}^{\prime}$.

Proof. Write $q=\left(c ; m ;\left(q_{i}\right)_{i \in H}\right), H=\{a, \ldots, h\}, \mathscr{D}=(\mathscr{S}, f), \mathscr{D}^{\prime}=$ $\left(\mathscr{S}^{\prime}, f^{\prime}\right), S_{1} \in \mathscr{S} \cup \mathscr{S}^{*}$ such that $a \in S_{1}, S_{1}^{\prime} \in \mathscr{S}^{\prime} \cup \mathscr{S}^{\prime *}$ such that $a \in S_{1}^{\prime}$, and

$$
\Phi_{Q}(q, \mathscr{D})=\left(c^{\prime} ; m^{\prime} ; \gamma_{a}, \gamma_{a+1}, \ldots\right)=\Phi_{Q}\left(q, \mathscr{D}^{\prime}\right) .
$$

Clearly, $S_{1} \in \mathscr{S} \Leftrightarrow c^{\prime}=c \Leftrightarrow S_{1}^{\prime} \in \mathscr{S}^{\prime}$ and if these conditions hold then $\max S_{1}=\min L(q)=\max S_{1}^{\prime}$, i.e., $S_{1}=S_{1}^{\prime}$.

In particular $(q, \mathscr{D})$ is minimal if and only if $\left(q, \mathscr{D}^{\prime}\right)$ is, and if they are minimal then $\mathscr{D}=\mathscr{D}^{\prime}$. So we assume $(q, \mathscr{D})$ is not minimal.

If $S_{1} \in \mathscr{S}^{*}$ then by above $S_{1}^{\prime} \in \mathscr{S}^{\prime *}$ as well. Define $A=\left\{i \in H \mid \gamma_{i} \neq q_{i}\right\}$; then we see that

$$
\max S_{1}= \begin{cases}-1+\min A, & \text { if } A \neq \varnothing, \\ h, & \text { if } A=\varnothing,\end{cases}
$$

and similarly for $S_{1}^{\prime}$, hence $S_{1}=S_{1}^{\prime}$.

Hence, in any case $S_{1}=S_{1}^{\prime}=\{a, \ldots, j\}$ for some $j \in H$. The reader will verify that $f(j)=f^{\prime}(j)$, which allows us to write $\operatorname{res}(q, \mathscr{D})=(\tilde{q}, \widetilde{\mathscr{D}})$ and $\operatorname{res}\left(q, \mathscr{D}^{\prime}\right)=\left(\tilde{q}, \widetilde{\mathscr{D}}^{\prime}\right)$, where $\tilde{q}$ is the same in the two cases. From the definition of $\Phi_{Q}$ it is easily seen that $\Phi_{Q}(\tilde{q}, \widetilde{\mathscr{D}})=\Phi_{Q}\left(\tilde{q}, \widetilde{\mathscr{D}}^{\prime}\right)$. So by induction we may assume that $\widetilde{\mathscr{D}}=\widetilde{\mathscr{D}}^{\prime}$. Hence $\operatorname{res}(q, \mathscr{D})=\operatorname{res}\left(q, \mathscr{D}^{\prime}\right)$ and the result follows from (2.3).

\section{Definitions 2.8 .}

1. An abstract characteristic sequence is a sequence

$$
C=\left(q_{0}, q_{1}, \ldots, q_{h}\right),
$$


such that $h \geq 1, q_{0}, q_{1} \in \mathbf{Z} \backslash\{0\}, q_{2}, \ldots, q_{h} \in \mathbf{N}$, and if we define $d_{i+1}=\operatorname{gcd}\left(q_{0}, \ldots, q_{i}\right)$ for $1 \leq i \leq h$, then $d_{2}>\cdots>d_{h+1}=1$.

2. If $C=\left(m, n, q_{2}, \ldots, q_{h}\right)$ is an abstract characteristic sequence and $d_{2}=\operatorname{gcd}(m, n)$, we let

$$
q(C)= \begin{cases}\left(d_{2} ; m ; n, q_{2}, \ldots, q_{h}\right), & \text { if }|m| \leq|n|, \\ \left(d_{2} ; n ; m, q_{2}, \ldots, q_{h}\right), & \text { if }|m|>|n|,\end{cases}
$$

which is a $Q$-sequence on $\{1, \ldots, h\}$. We also define

$$
\operatorname{Part}(C)=\{(\mathscr{S}, f) \in \operatorname{Part}(q(C))|1 \in \bigcup \mathscr{S} \Leftrightarrow| m|\leq| n \mid\} .
$$

Given $\mathscr{D} \in \operatorname{Part}(C)$, let

$$
\Phi(C, \mathscr{D})=\left(m, p n, q_{2}^{\prime}, \ldots, q_{h^{\prime}}^{\prime}\right),
$$

where $q_{2}^{\prime}, \ldots, q_{h^{\prime}}^{\prime}$ are given by $\Phi_{Q}(q(C), \mathscr{D})=\left(c^{\prime} ; m^{\prime} ; q_{1}^{\prime}, \ldots, q_{h^{\prime}}^{\prime}\right)$. Since the gcd's of $\Phi(C, \mathscr{D})$ are the same as those of $\Phi_{Q}(q(C), \mathscr{D})$, it follows from (2.6) that $\Phi(C, \mathscr{D})$ is an abstract characteristic sequence.

Lemma 2.9. If $C$ is an abstract characteristic sequence and $\mathscr{D}, \mathscr{D}^{\prime} \in \operatorname{Part}(C)$ are such that $\Phi(C, \mathscr{D})=\Phi\left(C, \mathscr{D}^{\prime}\right)$ then $\mathscr{D}=\mathscr{D}^{\prime}$.

Proof. This follows immediately from (2.7).

\section{THE THEOREM}

The aim of this section is to prove the main result of this paper:

\section{Theorem 3.1.}

1. Let $x, y \in \mathbf{k}((t))^{*}$ be series with nonzero orders and such that $\mathbf{k}\left(x, y^{p}\right)$ is dense in $\mathbf{k}((t))$. Then $C=[x, y]$ is an abstract characteristic sequence and there is a unique $\mathscr{D} \in \operatorname{Part}(C)$ such that $\left[x, y^{p}\right]=\Phi(C, \mathscr{D})$.

2. Let $C$ be an abstract characteristic sequence and $\mathscr{D} \in \operatorname{Part}(C)$. Then there exist $x, y \in \mathbf{k}((t))^{*}$ with nonzero orders such that $[x, y]=C$ and $\left[x, y^{p}\right]=\Phi(C, \mathscr{D})$. Note that $\mathbf{k}\left(x, y^{p}\right)$ must then be dense in $\mathbf{k}((t))$.

The reader may verify that the theorem is an easy consequence of the following two propositions:

Proposition 3.2. Suppose $x, y \in \mathbf{k}((t))^{*}$, ord $x=m \neq 0$, ord $y=n \neq 0$ and $|m| \leq|n|$, and let $c \in \mathbf{N}$ be such that $c \mid \operatorname{gcd}(m, n)$ and $|c|=|m|$. Let $[x, y: c]=\left(q_{1}, \ldots, q_{h}\right)$ and assume that $\operatorname{gcd}\left(c, q_{1}, \ldots, q_{h}\right)=1$. Then $q=$ $\left(c ; m ; q_{1}, \ldots, q_{h}\right)$ is a $Q$-sequence and the following hold.

1. Let $\left[x, y^{p}: c\right]=\left(q_{1}^{\prime}, \ldots, q_{h^{\prime}}^{\prime}\right)$ and suppose that $\operatorname{gcd}\left(c, q_{1}^{\prime}, \ldots, q_{h^{\prime}}^{\prime}\right)=$ 1. Then $\Phi_{Q}(q, \mathscr{D})=\left(c ; m ; q_{1}^{\prime}, \ldots, q_{h^{\prime}}^{\prime}\right)$ for a unique $\mathscr{D} \in \operatorname{Part}(q)$. Moreover, $1 \in \bigcup \mathscr{S}$, where $\mathscr{D}=(\mathscr{S}, f)$.

2. Suppose $|m|<|n|$, let $\left[x^{p}, y: p c\right]=\left(q_{1}^{\prime}, \ldots, q_{h^{\prime}}^{\prime}\right)$ and suppose that $\operatorname{gcd}\left(p c, q_{1}^{\prime}, \ldots, q_{h^{\prime}}^{\prime}\right)=1$. Then $\Phi_{Q}(q, \mathscr{D})=\left(p c ; p m ; q_{1}^{\prime}, \ldots, q_{h^{\prime}}^{\prime}\right)$ for a unique $\mathscr{D} \in \operatorname{Part}(q)$. Moreover, $1 \in \bigcup \mathscr{S}^{*}$, where $\mathscr{D}=(\mathscr{S}, f)$. 
Proposition 3.3. Let $q=\left(c ; m ; q_{1}, \ldots, q_{h}\right)$ be a $Q$-sequence and let $\mathscr{D}=$ $(\mathscr{S}, f) \in \operatorname{Part}(q)$. Define $q_{1}^{\prime}, \ldots, q_{h^{\prime}}^{\prime}$ by $\Phi_{Q}(q, \mathscr{D})=\left(c^{\prime} ; m^{\prime} ; q_{1}^{\prime}, \ldots, q_{h^{\prime}}^{\prime}\right)$. Then there exist $x, y \in \mathbf{k}((t))^{*}$ such that ord $x=m,[x, y: c]=\left(q_{1}, \ldots, q_{h}\right)$ and

$$
\left(q_{1}^{\prime}, \ldots, q_{h^{\prime}}^{\prime}\right)= \begin{cases}{\left[x, y^{p}: c\right],} & \text { if } 1 \in \bigcup \mathscr{S}, \\ {\left[x^{p}, y: p c\right],} & \text { if } 1 \in \bigcup \mathscr{S}^{*} .\end{cases}
$$

For the proof of these propositions, the following three lemmas are needed. The first two are easily verified.

Lemma 3.4. Let $S \subset \mathbf{Z}$ be nonempty and bounded below, $c \in \mathbf{N}, s=S(p|c|)$, and suppose that $\min S<s$. Let

$$
S^{-}=\{i \in S \mid i<s\} \text { and } S^{+}=\{i \in S \mid i \geq s\} .
$$

Write $\langle S ; c\rangle=\left(q_{1}, \ldots, q_{j_{0}-1}\right), m_{i}=q_{1}+\cdots+q_{i}$ for $1 \leq i<j_{0}$ and let $j=\max \left\{i \mid m_{i} \leq s\right\}$. Then

$$
\left\langle S^{-} ; p c\right\rangle= \begin{cases}\left(q_{1}, \ldots, q_{j-1}\right), & \text { if } s=m_{j}, \\ \left(q_{1}, \ldots, q_{j}\right), & \text { if } s>m_{j} .\end{cases}
$$

Moreover, if $s<\infty$ then $\left\langle S^{+} ; d_{j+1}\right\rangle=\left(s, m_{j+1}-s, q_{j+2}, \ldots, q_{j_{0}-1}\right)$, where $d_{j+1}=\operatorname{gcd}\left(c, q_{1}, \ldots, q_{j}\right)$.

Lemma 3.5. Let $S \subset \mathbf{Z}$ be nonempty and bounded below, $c \in \mathbf{N}, s=S(p|c|)$, and suppose that $\min S<s<\infty$ and $p \mid s$. Let

$$
S^{-}=\{i / p \mid i \in S \text { and } i<s\} \text { and } S^{+}=\{i \in S \mid i \geq s\}
$$

and define $q_{1}, \ldots, q_{j}, d_{2}, \ldots, d_{j+1}, Q_{2}, \ldots, Q_{i-1}$ by

$$
\begin{aligned}
\left\langle S^{-} ; c\right\rangle & =\left(q_{1}, \ldots, q_{j-1}\right), \\
q_{j} & =s / p-\left(q_{1}+\cdots+q_{j-1}\right), \\
d_{i+1} & =\operatorname{gcd}\left(c, q_{1}, \ldots, q_{i}\right), \\
\left\langle S^{+} ; p d_{j+1}\right\rangle & =\left(s, Q_{2}, \ldots, Q_{i-1}\right) .
\end{aligned}
$$

Then

$$
\langle S ; c\rangle= \begin{cases}\left(p q_{1}, \ldots, p q_{j-1}, p q_{j}+Q_{2}, Q_{3}, \ldots, Q_{i-1}\right), & \text { if } d_{j} / d_{j+1}=p, \\ \left(p q_{1}, \ldots, p q_{j}, Q_{2}, \ldots, Q_{i-1}\right), & \text { else. }\end{cases}
$$

Lemma 3.6. Suppose $x, y \in \mathbf{k}((t))^{*}$, ord $x=m \neq 0$, ord $y=n \neq 0$ and $c \in \mathbf{N}$ is such that $c \mid \operatorname{gcd}(m, n)$ and $|c|=|m|$. Let $[x, y: c]=\left(q_{1}, \ldots, q_{h}\right)$, $d_{i+1}=\operatorname{gcd}\left(c, q_{1}, \ldots, q_{i}\right)$ and $m_{i}=q_{1}+\cdots+q_{i}$ for $1 \leq i \leq h$, and let

$$
y=\sum_{i \geq n /|m|} a_{i} x_{1}^{i}+\rho
$$


be an expression satisfying the conditions of (1.1.3). Then the following hold.

1. Suppose $\rho \neq 0$, define $j$ by $\left\langle|m| \operatorname{Supp}_{x_{1}}(y-\rho) ; c\right\rangle=\left(q_{1}, \ldots, q_{j-1}\right)$ and $Q_{2}, \ldots, Q_{T}$ by $\left[\rho^{p}, x: p d_{j+1}\right]=\left(m, Q_{2}, \ldots, Q_{T}\right)$. Then

$$
\left[x, y^{p}: c\right]= \begin{cases}\left(p q_{1}, \ldots, p q_{j-1}, p q_{j}+Q_{2}, Q_{3}, \ldots, Q_{T}\right), & \text { if } d_{j} / d_{j+1}=p, \\ \left(p q_{1}, \ldots, p q_{j}, Q_{2}, \ldots, Q_{T}\right), & \text { else. }\end{cases}
$$

2. If $|m|<|n|$, let

$$
\begin{aligned}
s_{0} & =\left(\operatorname{Supp}_{x_{1}}(y-\rho)\right)(p), \\
s & = \begin{cases}|m| s_{0}, & \text { if } s_{0}<\infty, \\
\operatorname{ord} \rho, & \text { if } s_{0}=\infty,\end{cases} \\
y & =\sum_{i<s_{0}} a_{i}\left(x_{1}^{p}\right)^{i / p}+\tilde{\rho}, \\
\tilde{\rho} & =\sum_{i \geq s_{0}} a_{i} x_{1}^{i}+\rho .
\end{aligned}
$$

Assume that $\tilde{\rho} \neq 0$ and note that ord $\tilde{\rho}=s<\infty$. Then

$$
\begin{aligned}
{\left[x^{p}, y: p c\right] } & =\left(q_{1}, \ldots, q_{j}, \widehat{s-m_{j}}, Q_{2}, \ldots, Q_{T}\right), \\
{\left[\tilde{\rho}, x: d_{j+1}\right] } & =\left(m, m_{j+1}-s, q_{j+2}, \ldots, q_{h}\right),
\end{aligned}
$$

where $j=\max \left\{i \mid m_{i} \leq s\right\}$ and where $Q_{2}, \ldots, Q_{T}$ are defined by $\left[\tilde{\rho}, x^{p}: d_{j+1}\right]=\left(p m, Q_{2}, \ldots, Q_{T}\right)$.

Proof. For the first assertion, consider $y^{p}=\sum a_{i}^{p} x_{1}^{p i}+\rho^{p}$. If $\mid$ ord $\rho^{p}|<| m \mid$ then that expression for $y^{p}$ satisfies the conditions of (1.1.3) and it follows immediately that

$$
\left[x, y^{p}: c\right]=\left(p q_{1}, \ldots, p q_{j}, Q_{2}, \ldots, Q_{T}\right),
$$

which is the desired result since $d_{j} / d_{j+1} \neq p$.

If $\mid$ ord $\rho^{p}|=| m \mid$ then let $\rho^{p}=\sum \alpha_{i} x_{1}^{i}+\omega$ be an expression satisfying the conditions of (1.1.3). By the inversion theorem (1.2), $\left[x, \rho^{p}: p d_{j+1}\right]=$ ( $p$ ord $\rho, Q_{2}, \ldots, Q_{T}$ ), so we can write for some $i$

$$
\left\langle|m| \operatorname{Supp}_{x_{1}}\left(\rho^{p}-\omega\right) ; p d_{j+1}\right\rangle=\left(p \operatorname{ord} \rho, Q_{2}, \ldots, Q_{i-1}\right),
$$

and if $\omega \neq 0$ then

$$
\begin{aligned}
Q_{i} & =\operatorname{ord} \omega-\left(p \operatorname{ord} \rho+Q_{2}+\cdots+Q_{i-1}\right), \\
\gamma & =\operatorname{gcd}\left(p d_{j+1}, p \operatorname{ord} \rho, Q_{2}, \ldots, Q_{i}\right), \\
{[\omega, x: \gamma] } & =\left(m, Q_{i+1}, \ldots, Q_{T}\right) .
\end{aligned}
$$

Then by (3.5), with $S=|m| \operatorname{Supp}_{x_{1}}\left(y^{p}-\omega\right)$ and $s=\operatorname{ord} \rho^{p}$,

$$
\langle S ; c\rangle= \begin{cases}\left(p q_{1}, \ldots, p q_{j-1}, p q_{j}+Q_{2}, Q_{3}, \ldots, Q_{i-1}\right), & \text { if } d_{j} / d_{j+1}=p, \\ \left(p q_{1}, \ldots, p q_{j}, Q_{2}, \ldots, Q_{i-1}\right), & \text { else, }\end{cases}
$$

and the rest is easily verified. 
For the second assertion, define $j_{0}$ by

$$
\left\langle|m| \operatorname{Supp}_{x_{1}}(y-\rho) ; c\right\rangle=\left(q_{1}, \ldots, q_{j_{0}-1}\right),
$$

and note that $j \leq j_{0}$. If $s_{0}=\infty$ then $j=j_{0}, \tilde{\rho}=\rho, s=m_{j_{0}}$ and since $y=\sum a_{i}\left(x_{1}^{p}\right)^{i / p}+\rho$ satisfies the conditions of (1.1.3),

$$
\begin{aligned}
{\left[x^{p}, y: p c\right] } & =\left(q_{1}, \ldots, q_{j_{0}}, Q_{2}, \ldots, Q_{T}\right), \\
{\left[\tilde{\rho}, x: d_{j+1}\right] } & =\left[\rho, x, d_{j_{0}+1}\right]=\left(m, q_{j_{0}+1}, \ldots, q_{h}\right),
\end{aligned}
$$

as desired. If $s_{0}<\infty$ then $j<j_{0}$ and $|s|=|m|$. Let $S=|m| \operatorname{Supp}_{x_{1}}(y-\rho)$, then $S(p|c|)=s<\infty$ and by (3.4) [x $\left.x^{p}, y: p c\right]$ begins with

$$
\begin{aligned}
\left\langle p|m| \operatorname{Supp}_{x_{1}^{p}}(y-\tilde{\rho}) ; p c\right\rangle & =\langle\{i \in S \mid i<s\} ; p c\rangle \\
& = \begin{cases}\left(q_{1}, \ldots, q_{j-1}\right), & \text { if } s=m_{j}, \\
\left(q_{1}, \ldots, q_{j}\right), & \text { if } s<m_{j} .\end{cases}
\end{aligned}
$$

The next term of $\left[x^{p}, y ; p c\right]$ is ord $\tilde{\rho}-m_{j-1}=s-m_{j-1}=q_{j}$ if $s=m_{j}$, or ord $\tilde{\rho}-m_{j}=s-m_{j}$ if $s>m_{j}$, and the gcd of $p c$ and all those terms is $\operatorname{gcd}\left(p c, q_{1}, \ldots, q_{j}, s-m_{j}\right)=d_{j+1}$ in any case. Hence

$$
\left[x^{p}, y: p c\right]=\left(q_{1}, \ldots, q_{j}, \widehat{s-m_{j}}, Q_{2}, \ldots, Q_{T}\right),
$$

as desired. By (3.4) again,

$$
\left[x, \tilde{\rho}: d_{j+1}\right]=\left(s, m_{j+1}-s, q_{j+2}, \ldots, q_{h}\right),
$$

and the result follows from the Inversion Theorem 1.2.

Proof of (3.2). Uniqueness of $\mathscr{D}$ is clear by (2.7). Given an integer $\nu \geq 0$ consider the statement:

$\left(1_{\nu}\right) \quad$ The assertion (1) of the proposition holds whenever $|\operatorname{ord} x| \leq p^{\nu}$.

We define $\left(2_{\nu}\right)$ in a similar fashion. We shall prove that $\left(1_{0}\right)$ holds and that $\left(1_{\nu}\right) \Rightarrow\left(2_{\nu}\right) \Rightarrow\left(1_{\nu+1}\right)$ for $\nu \geq 0$.

Let $d_{2}, \ldots, d_{h+1}$ be the gcd's of $q$, let $m_{i}=q_{1}+\cdots+q_{i}$ for $1 \leq i \leq h$, and let

$$
y=\sum_{i \geq n /|m|} a_{i} x_{1}^{i}+\rho
$$

be an expression satisfying the conditions of (1.1.3). Define $j_{0}$ by

$$
\left\langle|m| \operatorname{Supp}_{x_{1}}(y-\rho) ; c\right\rangle=\left(q_{1}, \ldots, q_{j_{0}-1}\right) .
$$

So if $\rho \neq 0$ then $q_{j_{0}}=\operatorname{ord} \rho-\left(q_{1}+\cdots+q_{j_{0}-1}\right)$, $\mid$ ord $\rho|=| d_{j_{0}+1} \mid$ and

$$
\left[\rho, x: d_{j_{0}+1}\right]=\left(m, q_{j_{0}+1}, \ldots, q_{h}\right) .
$$

Proof of $\left(1_{0}\right)$ : Assume $|m|=1$, then $\rho=0$ and

$$
\left[x, y^{p}: c\right]=\left\langle p \operatorname{Supp}_{x_{1}} y ; c\right\rangle=\left(p q_{1}, \ldots, p q_{h}\right) \text {. }
$$


Let $\mathscr{S}=\{H\}$, let $f: H \rightarrow \mathbf{Z}$ be the zero function and $\mathscr{D}=(\mathscr{S}, f)$. Then $\mathscr{D} \in \operatorname{Part}(q), 1 \in \bigcup \mathscr{S}$ and $\Phi_{Q}(q, \mathscr{D})=\left(c ; m ; p q_{1}, \ldots, p q_{h}\right)$, as desired. Note that $(q, \mathscr{D})$ is minimal.

Proof that $\left(1_{\nu}\right) \Rightarrow\left(2_{\nu}\right)$ : Assume that $|m|=p^{\nu}$ and define $s_{0}, s, \tilde{\rho}$ and $j$ as in the second assertion of (3.6). Note that $\tilde{\rho} \neq 0$ and that ord $\tilde{\rho}=s$ (otherwise $s_{0}=\infty$ and $\rho=0, y=\sum a_{i}\left(x_{1}^{p}\right)^{i / p}$, thus $\left(q_{1}^{\prime}, \ldots, q_{h^{\prime}}^{\prime}\right)=\left[x^{p}, y: p c\right]=$ $\left\langle|m| \operatorname{Supp}_{x_{1}} y ; p c\right\rangle=\left(q_{1}, \ldots, q_{h}\right)$, whence $\operatorname{gcd}\left(p c, q_{1}^{\prime}, \ldots, q_{h^{\prime}}^{\prime}\right)=p$, contradiction). Hence by (3.6),

$$
\begin{aligned}
{\left[x^{p}, y: p c\right] } & =\left(q_{1}, \ldots, q_{j}, \hat{\alpha}, Q_{2}, \ldots, Q_{T}\right), \\
{\left[\tilde{\rho}, x: d_{j+1}\right] } & =\left(m, q_{j+1}-\alpha, q_{j+2}, \ldots, q_{h}\right),
\end{aligned}
$$

where $\alpha=s-m_{j}$ and $Q_{2}, \ldots, Q_{T}$ are defined by

$$
\left[\tilde{\rho}, x^{p}: d_{j+1}\right]=\left(p m, Q_{2}, \ldots, Q_{T}\right) \text {. }
$$

Note that $\operatorname{gcd}\left(d_{j+1}, p m, Q_{2}, \ldots, Q_{T}\right)=\operatorname{gcd}\left(p c, q_{1}^{\prime}, \ldots, q_{h^{\prime}}^{\prime}\right)=1$, and that

$$
\tilde{q}=\left(d_{j+1} ; s ; m, q_{j+1}-\alpha, q_{j+2}, \ldots, q_{h}\right)
$$

is a restriction of type 2 of $q$. So, by $\left(1_{\nu}\right)$ applied to $(\tilde{\rho}, x)$, there exists $\widetilde{\mathscr{D}}=(\widetilde{\mathscr{S}}, \tilde{f}) \in \operatorname{Part}(\tilde{q})$ such that $j \in \bigcup \widetilde{\mathscr{S}}$ and

$$
\Phi_{Q}(\tilde{q}, \widetilde{\mathscr{D}})=\left(d_{j+1} ; s ; p m, Q_{2}, \ldots, Q_{T}\right) .
$$

By (2.3), there exists $\mathscr{D}=(\mathscr{S}, f) \in \operatorname{Part}(q)$ such that $\operatorname{res}(q, \mathscr{D})=(\tilde{q}, \widetilde{\mathscr{D}})$. Let $S_{1}=\{1, \ldots, j\}$ then $S_{1} \in \mathscr{S}^{*}$ and by definition of $\Phi_{Q}$ we have

$$
\Phi_{Q}(q, \mathscr{D})=\left(p c ; p m ; q_{1}, \ldots, q_{j}, \widehat{f(j)}, Q_{2}, \ldots, Q_{T}\right),
$$

which is the desired result since $f(j)=\alpha$.

Proof that $\left(2_{\nu}\right) \Rightarrow\left(1_{\nu+1}\right)$. Assume $|m|=p^{\nu+1}$. Then $\rho \neq 0$, for otherwise $p^{\nu+1} \mid \operatorname{gcd}\left(c, q_{1}, \ldots, q_{h}\right)=1$. Let $j=j_{0}$, note that

$$
\tilde{q}=\left(d_{j+1} ; \text { ord } \rho ; m, q_{j+1}, \ldots, q_{h}\right)
$$

is the restriction of type 1 of $q$ and define $Q_{2}, \ldots, Q_{T}$ by $\left[\rho^{p}, x: p d_{j+1}\right]=$ $\left(m, Q_{2}, \ldots, Q_{T}\right)$. By (3.6),

$$
\left[x, y^{p}: c\right]= \begin{cases}\left(p q_{1}, \ldots, p q_{j-1}, p q_{j}+Q_{2}, Q_{3}, \ldots, Q_{T}\right), & \text { if } d_{j} / d_{j+1}=p, \\ \left(p q_{1}, \ldots, p q_{j}, Q_{2}, \ldots, Q_{T}\right), & \text { else. }\end{cases}
$$

Since $\operatorname{gcd}\left(c, p q_{1}, \ldots, p q_{j}\right)=p d_{j+1}$, it follows that

$$
\operatorname{gcd}\left(p d_{j+1}, Q_{2}, \ldots, Q_{T}\right)=\operatorname{gcd}\left(c, q_{1}^{\prime}, \ldots, q_{h^{\prime}}^{\prime}\right)=1 .
$$

Hence by $\left(2_{\nu}\right)$ applied to $(\rho, x)$ there exists $\widetilde{\mathscr{D}}=(\widetilde{\mathscr{S}}, \tilde{f}) \in \operatorname{Part}(\tilde{q})$ such that $j \in \bigcup \widetilde{\mathscr{S}}^{*}$ and

$$
\Phi_{Q}(\tilde{q}, \widetilde{\mathscr{D}})=\left(p d_{j+1} ; p \operatorname{ord} \rho ; m, Q_{2}, \ldots, Q_{T}\right)
$$


By (2.3) there exists $\mathscr{D}=(\mathscr{S}, f) \in \operatorname{Part}(q)$ such that $\operatorname{res}(q, \mathscr{D})=(\tilde{q}, \widetilde{\mathscr{D}})$. Let $S_{1}=\{1, \ldots, j\}$, then $S_{1} \in \mathscr{S}$ and by definition of $\Phi_{Q}$ we have

$$
\Phi_{Q}(q, \mathscr{D})=\left\{\begin{array}{c}
\left(c ; m ; p q_{1}, \ldots, p q_{j-1}, p q_{j}+Q_{2}, Q_{3}, \ldots, Q_{T}\right), \\
\text { if } d_{j} / d_{j+1}=p, \\
\left(c ; m ; p q_{1}, \ldots, p q_{j}, Q_{2}, \ldots, Q_{T}\right), \quad \text { else, }
\end{array}\right.
$$

which is the desired result.

Proof of (3.3). If $(q, \mathscr{D})$ is minimal then

$$
x=t^{m}, \quad y=\sum_{i=1}^{h} t^{q_{1}+\cdots+q_{i}}
$$

satisfy the requirements. Suppose $(q, \mathscr{D})$ is not minimal and let $(\tilde{q}, \widetilde{D})=$ $\operatorname{res}(q, \mathscr{D}), \tilde{q}=\left(d_{j+1} ; \ldots\right)$ and $S_{1}=\{1, \ldots, j\}$. We may assume that the result holds for $(\tilde{q}, \widetilde{\mathscr{D}})$.

Case $S_{1} \in \mathscr{S}$. Then $\tilde{q}=\left(d_{j+1} ; q_{1}+\cdots+q_{j} ; m, q_{j+1}, \ldots, q_{h}\right)$ is the restriction of type 1 of $q$. Define $Q_{2}, \ldots, Q_{T}$ by

$$
\Phi_{Q}(\tilde{q}, \widetilde{D})=\left(p d_{j+1} ; p\left(q_{1}+\cdots+q_{j}\right) ; m, Q_{2}, \ldots, Q_{T}\right) .
$$

Since the result holds for $(\tilde{q}, \widetilde{D})$, there are $\rho, x \in \mathbf{k}((t))^{*}$ with ord $\rho=q_{1}+$ $\cdots+q_{j},\left[\rho, x: d_{j+1}\right]=\left(m, q_{j+1}, \ldots, q_{h}\right)$ and $\left[\rho^{p}, x: p d_{j+1}\right]=\left(m, Q_{2}, \ldots\right.$, $\left.Q_{T}\right)$. Now let $x_{1}$ be an $m_{0}$ th root of $x$ and let

$$
y=\sum_{i=1}^{j-1} x_{1}^{\left(q_{1}+\cdots+q_{i}\right) /|m|}+\rho .
$$

Since $\mid$ ord $\rho|<| m \mid$ it is clear that $[x, y: c]=\left(q_{1}, \ldots, q_{h}\right)$. By (3.6),

$$
\left[x, y^{p}: c\right]= \begin{cases}\left(p q_{1}, \ldots, p q_{j-1}, p q_{j}+Q_{2}, Q_{3}, \ldots, Q_{T}\right), & \text { if } d_{j} / d_{j+1}=p, \\ \left(p q_{1}, \ldots, p q_{j}, Q_{2}, \ldots, Q_{T}\right), & \text { else. }\end{cases}
$$

By definition of $\Phi_{Q},\left[x, y^{p}: c\right]=\left(q_{1}^{\prime}, \ldots, q_{h^{\prime}}^{\prime}\right)$.

Case $S_{1} \in \mathscr{S}^{*}$. Then $\tilde{q}=\left(d_{j+1} ; q_{1}+\cdots+q_{j}+f(j) ; m, q_{j+1}-f(j), q_{j+2}, \ldots\right.$, $q_{h}$ ) is a restriction of type 2 of $q$. Define $Q_{2}, \ldots, Q_{T}$ by

$$
\Phi_{Q}(\tilde{q}, \widetilde{\mathscr{D}})=\left(d_{j+1} ; q_{1}+\cdots+q_{j}+f(j) ; p m, Q_{2}, \ldots, Q_{T}\right) .
$$

Since the result holds for $(\tilde{q}, \widetilde{\mathscr{D}})$, there exist $\tilde{\rho}, x \in \mathbf{k}((t))^{*}$ such that ord $\tilde{\rho}=$ $q_{1}+\cdots+q_{j}+f(j),\left[\tilde{\rho}, x: d_{j+1}\right]=\left(m, q_{j+1}-f(j), q_{j+2}, \ldots, q_{h}\right)$ and $\left[\tilde{\rho}, x^{p}: d_{j+1}\right]=\left(p m, Q_{2}, \ldots, Q_{T}\right)$. Let $x_{1}$ be an $m_{0}$ th root of $x$ and define

$$
\begin{array}{ll}
y=\sum_{i=1}^{j-1} x_{1}^{\left(q_{1}+\cdots+q_{i}\right) /|m|}+\tilde{\rho}, & \text { if } f(j)=0, \\
y=\sum_{i=1}^{j} x_{1}^{\left(q_{1}+\cdots+q_{i}\right) /|m|}+\tilde{\rho}, & \text { if } f(j)>0 .
\end{array}
$$


We claim that $[x, y: c]=\left(q_{1}, \ldots, q_{h}\right)$. In fact, this is clear if $\mid$ ord $\tilde{\rho}|<| m \mid$ (in which case $f(j)=0$ ). If $\mid$ ord $\tilde{\rho}|=| m \mid$ then by the inversion theorem

$$
\left[x, \tilde{\rho}: d_{j+1}\right]=\left(q_{1}+\cdots+q_{j}+f(j), q_{j+1}-f(j), q_{j+2}, \ldots, q_{h}\right),
$$

and the claim easily follows. Now by (3.6),

$$
\left[x^{p}, y: p c\right]=\left(q_{1}, \ldots, q_{j}, \widehat{f(j)}, Q_{2}, \ldots, Q_{T}\right) .
$$

Hence by definition of $\Phi_{Q}$ we have $\left[x^{p}, y: p c\right]=\left(q_{1}^{\prime}, \ldots, q_{h^{\prime}}^{\prime}\right)$, as desired.

\section{REFERENCES}

1. S. Abhyankar and T. T. Moh, Embeddings of the line in the plane, J. Reine Angew. Math. 276 (1975), 148-166.

2. R. Ganong, Kodaira dimension of embeddings of the line in the plane, J. Math. Kyoto Univ. 25 (1985), 649-657.

3. T. T. Moh, On characteristic pairs of algebroid plane curves for characteristic $p$, Bull. Math. Academia Sinica 1 (1973), 75-91.

4. __ On the classification problem of embedded lines in characteristic p, Algebraic Geometry and Commutative Algebra in Honor of Masayoshi Nagata, 1987, pp. 267-279.

5. M. Nagata, $A$ theorem of Gutwirth, J. Math. Kyoto Univ. 11 (1971), 149-154.

6. K. P. Russell, Hamburger-Noether expansions and approximate roots of polynomials, Manuscripta Math. 31 (1980), 25-95. 0027

Department of Mathematics, University of Kentucky, LeXington, Kentucky 40506-

Current address: Department of Mathematics, University of Ottawa, Ottawa, Canada K1N 6N5 